\title{
LOW BACK PAIN IN PHYSIOTHERAPY STUDENTS: PREVALENCE AND THE ASSOCIATION WITH NEUROMUSCULAR FINDINGS
}

\begin{abstract}
Background: Physiotherapy students are prone to low back pain (LBP) due to studying and their active involvement in clinical treatment of patients. As a result of pathology, muscle activity is influenced, affecting optimal function of the spine.

Method: Physiotherapy students enrolled for 2010 at the University of the Witwatersrand participated in a cross-sectional study. A questionnaire and physical assessment were completed.

Results: The study revealed that the lifetime LBP prevalence was $36 \%$ among physiotherapy students. Associations with LBP were hours of practical exposure, posterior-anterior mobilisations on L4 $(p=0.003)$ and L5 ( $p \leq 0.001)$ centrally and unilaterally, left lumbar multifidus (LM) cross-sectional area $(p=0.02)$, right obliquus internus abdominis (OI) $(p=0.02)$ and right transversus abdominis $(\operatorname{Tr} A)$ thickness at rest $(p=0.02)$, as well as the pull of the TrA during contraction on the left $(p=0.03)$.
\end{abstract}

Discussion: Hours of practical exposure may play a role in lumbar pathology. Due to pathology, muscle imbalances of LM, TrA and OI affect the stability of the spine which may lead to recurrences.

Conclusion: Practical exposure as well as LM, TrA and OI muscle imbalances were associated with LBP in physiotherapy students. Awareness of the factors associated with LBP while studying at an undergraduate level may lead to better prevention of $L B P$.

\section{KEY WORDS: LOW BACK PAIN, PHYSIOTHERAPY, ABDOMINAL MUSCLE THICKNESS, RECRUITMENT}

\section{INTRODUCTION}

Physiotherapy students are prone to low back pain (LBP) due to a flexion posture while studying, lifting patients and working in incorrect positions. A study on physiotherapy students conducted at an Australian tertiary institution found a $69 \%$ lifetime prevalence of LBP (Nyland and Grimmer 2003). Of the students, $65 \%$ experienced LBP in

\section{Correspondence Author:}

Elaine Burger

Department of Physiotherapy,

Faculty of Health Sciences,

University of the Witwatersrand,

7 York Road

Parktown 2193

Johannesburg, South Africa

Email: burger.elaine@gmail.com the preceding 12-months, compared to $44 \%$ in the preceding month and $28 \%$ in the preceding week. The prevalence of LBP was particularly high in final year students, and students between 20 and 21 years of age (Nyland and Grimmer 2003). The onset of LBP amongst young physiotherapists occurs between the ages of 21 and 30 years and within the first four years of qualification and starting to practice physiotherapy, while the prevalence of LBP increased from first year to fourth year (Karachi et al 2007).

Factors associated with LBP among physiotherapy students include students' exposure to educational activities such as "sitting and looking down" while studying, and clinical treatment of patients (Nyland and Grimmer 2003). The length of study years of physiotherapy students (years two to

\author{
Burger E, MSc Physiotherapy'; \\ Myezwa H, PhD'; \\ Naidoo V, MSc Physiotherapy'; \\ Olivier B, MSc Physiotherapy'; \\ Rothberg A, PhD ${ }^{2}$ \\ Department of Physiotherapy, \\ Faculty of Health Sciences, \\ University of the Witwatersrand. \\ Faculty of Health Sciences, \\ University of the Witwatersrand.
}


abdominis (TrA) and lumbar multifidus (LM) (Comerford and Mottram 2001b). Furthermore, segmental reflex inhibition of the LM and TrA, a decrease of the cross-sectional area (CSA), atrophy, altered recruitment patterns and timing occur at the affected level in individuals with LBP (Comerford and Mottram 2001a, Comerford and Mottram 2001b). This predisposes to recurrence of pain and progression of these muscle imbalances and dysfunction (Hides et al 1996). A physiotherapy student who has experienced LBP may mitigate further damage and predisposition to LBP by addressing these specific muscles during training before qualifying and starting work as a physiotherapist.

Therefore the aim of this study was to establish the prevalence of LBP and neuromuscular mechanisms associated with LBP among undergraduate physiotherapy students.

\section{METHODS}

\section{Study design}

This was a cross-sectional, descriptive study.

\section{Population and sampling}

The population consisted of physiotherapy students registered for the 2010 academic year at the University of the Witwatersrand and all students $(n=208)$ were invited via class announcements to participate in the study. Participants presenting with: scoliosis or any struc- tural deformity; who had undergone skeletal, muscular or ligamentous surgery in the previous six months; who used back, leg or neck braces; who suffer from known diseases such as cancer, tuberculosis, lung diseases or AIDS, or who were pregnant, were excluded. LBP was defined as an "ache, pain or discomfort in the lower back that lasted for more than 24 hours when present" (Nyland and Grimmer 2003). Students were tested at a time before or after their scheduled lectures at a venue in the Physiotherapy Department. The ethical considerations of confidentiality and anonymity were maintained throughout the data collection and analysis of the questionnaire and physical assessment tests. Ethical clearance was obtained from the Human Research Ethics Committee of the University of the Witwatersrand.

\section{Tools and measurements}

The data-collection process consisted of a questionnaire and physical assessment. Passive accessory intervertebral movements, TrA recruitment assessment as well as ultrasound imaging (USI) of segmental LM, TrA and Obliquus Internus Abdominus (OI) made up the physical assessment.

\section{Questionnaire}

The questionnaire was developed from the literature (Karachi et al 2007). Content validity was ensured through a focus group discussion that included clinical and academic experts in the field. All experts had more than seven years' experience and were in possession of a postgraduate qualification. To establish test-retest reliability, twelve third year occupational therapy students completed the questionnaire on two

Table 1: Repeatability of the Physical Tests $(n=13)$

\begin{tabular}{|l|c|c|}
\hline Physical tests & ICC & $95 \% \mathrm{CI}$ \\
\hline Recruitment of TrA & 0.65 & $0.20-0.88$ \\
\hline LM CSA Left by USI & 0.75 & $0.38-0.92$ \\
\hline LM CSA Right by USI & 0.72 & $0.32-0.90$ \\
\hline Right OI at rest by USI & 0.99 & $0.99-1.0$ \\
\hline Right OI contracted by USI & 0.99 & $0.98-1.0$ \\
\hline Right TrA at rest by USI & 0.99 & $0.99-1.0$ \\
\hline Right TrA contracted by USI & 0.99 & $0.98-1.0$ \\
\hline Right TrA slide by USI & 0.95 & $0.85-0.99$ \\
\hline
\end{tabular}

$\mathrm{Cl}=95 \%$ confidence interval, ICC = intra-class coefficient, LM = lumbar multifidus, $\mathrm{OI}=$ obliquus internus abdominis, $\operatorname{Tr} \mathrm{A}=$ transversus abdominis, USI = ultrasound imaging.

Table 2: Cumulative Hours Exposure at Time of Testing

\begin{tabular}{|c|c|c|c|c|c|c|}
\hline \multirow{2}{*}{ Students } & \multicolumn{3}{|c|}{$\begin{array}{l}\text { Actual hours exposure at time of testing in } \\
2010\end{array}$} & \multicolumn{3}{|c|}{$\begin{array}{l}\text { Cumulative exposure at time of test (actual } \\
\text { in } 2010 \text { plus total from prior years) }\end{array}$} \\
\hline & Theory & Practical & Total & Theory & Practical & Total \\
\hline First year $(n=57)$ & 436 & 0 & 436 & 436 & 0 & 436 \\
\hline Second year $(n=45)$ & 426 & 76 & 502 & 1007 & 76 & 1083 \\
\hline Third year $(n=41)$ & 306 & 563 & 869 & 1738 & 715 & 2453 \\
\hline Fourth year $(n=36)$ & 150 & 57 & 207 & 1990 & 959 & 2949 \\
\hline
\end{tabular}

To explain the above calculations, for example at the time of testing in 2010, the third years had 563 hours of practical exposure for the year. This was then added to the scheduled exposure/s in prior years, giving a cumulative exposure of 2453 hours. Although the fourth year students had the most hours' exposure cumulatively for theory and practical, they were assessed near the beginning of the year. They were therefore only exposed to 57 hours of practical and 150 hours of theory for the year, giving a cumulative figure of 2949 up to that point. 
separate occasions, one week apart. Each outcome variable was scored and compared with the first questionnaire completed for each student. Test-retest reliability ranged between $75 \%$ and $92 \%$ (Kappa) on the variables of the questionnaire. Furthermore, a pilot study was conducted on 45, fourth year physiotherapy students. Students that participated in the pilot study were not included in the main study. The overall content of the questionnaire was satisfactory and only minor changes were made.

\section{Passive accessory intervertebral move- ments}

Postero-anterior (PA) passive accessory intervertebral movements (central and unilateral) on L4 and L5 were applied. The participant lay in prone with their arms next to their side and face turned to one side. The researcher stood at the left side of the participant. The central PA was performed as follows: the left hand, the part between the pisiform and the hook of the hamatum (of the ulnar border of the hand), was in contact with the spinous process of L4. The researcher's shoulders were directly over the vertebrae to be mobilised and the wrist of the left hand in full extension, forearm neutral (between supination and pronation) for optimal contact. The left hand was reinforced by the right. The unilateral PA was performed as follows: if assessing the left side, the researcher stands on left side of participant, placing thumbs on back adjacent to spinous process on the left. The thumbs are pointing towards each other, fingers spread around for stability. Pain was assessed using the four grades of movement (Maitland et al 2001). If pain was experienced on mobilisation it was recorded as 'yes', if no pain was experienced on mobilisation, it was recorded as 'no'. The grade of mobilisation was recorded when pain occurred.

\section{TrA recruitment assessment}

The participant was positioned in 'crook' lying. The first author palpated inferiorly and medially from the anterior superior iliac spine (ASIS) along the inguinal ligament and felt for muscle tensioning during the abdominal drawing-in manoeuvre (ADIM). The participant was instructed to hollow or draw in the lower abdominal wall without obliquus externus (OE) rib cage expansion, posterior pelvic tilt or OI bulge. Normal breathing while maintaining a consistent minimal contraction at 20-30\% maximal voluntary contraction (MVC) sustained for 15 seconds, procedure repeated twice and 'feeling easy', was considered ideal recruitment (Comerford and Mottram 2001a). Abnormal recruitment of $\operatorname{Tr} A$ was identified when substitution of surrounding muscles occurred.

\section{USI of segmental LM, TrA and OI}

A digital ultrasound diagnostic imaging system (Mindray DP-2200) was used in this study. A $5 \mathrm{MHz}$ curved array probe was used with the ultrasound machine in B mode (Stokes et al 2005). The first author obtained training for use of the USI machine. The CSA of LM was assessed according to the procedure described by Stokes et al (2005) which involved placing the probe longitudinally (sagittal plane) to identify L4. When L4 was identified, the probe was rotated $90^{\circ}$ for a transverse application. The CSA for each side was captured after the participant lifted the ipsilateral leg (Stokes et al 2005). A resting image of the left abdominal wall was captured with the participant in supine. The transducer was placed along the lateral abdominal wall, superior to the iliac crest, along the mid-axillary line (Teyhen et al 2009). TrA activation was assessed during the ADIM. Percentage change in thickness of TrA and OI was calculated and is a valid measure of activity compared to electromyography (Hodges et al 2003) especially during low levels of muscle contraction (up to $30 \%$ of MVC) (Hodges et al 2003).

The first author performed all tests and was blinded to LBP status of the participants. Intra-rater reliability was established for all physical tests during the pilot study (Table 1). The results of the measurement method comparison indicate an acceptable repeatability of all the measures.

\section{Data analysis}

The statistical analysis was conducted using Statistica ${ }^{\circledR}$ Version 10 (StatSoft
Inc, Tulsa, USA). The prevalence of LBP between the different year groups was compared using Pearson's chi square test. Neuromuscular associations with LBP were identified using Pearson's chi square or Fisher's exact tests for categorical data and student's t-test for continuous data where appropriate. Testing was done at the 0.05 level of significance. Muscle thickness was reported as absolute thickness at rest and thickness percentage change or muscle activity (TrA and OI). Thickness percentage change was calculated as muscle thickness during activity as a ratio to muscle thickness at rest (Hodges et al 2003), thus muscle thickness in contracted state minus muscle thickness at rest, divided by muscle thickness at rest, multiplied by 100 [TrA activity= (TrA contacted - TrA rest)/TrA rest x 100] (Teyhen et al 2009).

\section{RESULTS}

Of the total population of 208 students, 200 questionnaires were returned, of which six were incomplete; hence 194 (93\%) questionnaires were included in the analysis. One hundred and seventy nine $(86 \%)$ completed the physical examination. Of those who completed the questionnaires, thirty seven (19\%) were male and $157(81 \%)$ were female. The mean age was $20.2( \pm 2.1)$ years. The lifetime prevalence of LBP amongst the physiotherapy students was 36\% $(n=194)$. Figure 5 shows the prevalence of LBP at the time of testing of first to fourth years.

The students' actual exposure in cumulative hours at the time of testing per year is shown in Table 2. Notably in Table 3 the relationships between LBP and the cumulative practical exposure and total exposure hours were established $(\mathrm{p}=0.02$ and $\mathrm{p}=0.04$ respectively). Total exposure at the time of testing increased by $\pm 150 \%$ per year up to third year, but was less for the fourth years.

LBP was associated with the expression of pain on PA mobilisation of L4 $(p=0.003)$ and L5 $(p=<0.001)$. LBP was furthermore also associated with four neuromuscular variables namely the left (L) LM CSA ( $p=0.02$ ), the OI and TrA during rest on the right $(\mathrm{R})$ side $(\mathrm{p}=0.02 ; \mathrm{p}=0.02)$ and the $\mathrm{L} \operatorname{Tr} \mathrm{A}$ slide 
$(p=0.03)$. Figure 1 shows an image of the CSA of the L4 LM at rest. In Figure 2 the CSA of the left and right side of LM are seen as asymmetrical at rest. Figure 3 shows a resting image of the TrA while Figure 4 shows TrA activation during ADIM.

\section{DISCUSSION}

The present work is the first study to show measurements with ultrasound imaging of LM and TrA on physiotherapy students and adds to the specific knowledge of what anatomical changes take place as a result of exposure to training activities. The aim of this study was to establish the prevalence of LBP and neuromuscular mechanisms associated with LBP among undergraduate physiotherapy students. The major determinant of LBP in this study appeared to be the students' exposure to cumulative hours of practical work (Table 3), particularly over the first three years. In contrast to some of the findings of Nyland and Grimmer (2003), it did not appear that hours spent "sitting looking down" engaged in theoretical learning played a role. The unexpected and counter-intuitive finding of a reduced incidence of LBP in the fourth year students could be explained by the timing of assessments in that group (i.e. they were assessed after the end-of-year holidays, after only a few weeks of theoretical work and before starting clinical work).

The lifetime prevalence of LBP in this study sample of first-to-fourth year physiotherapy students was found to be $36 \%$ and similar to the average rate of $45 \%$ calculated from studies cited in the literature (Falavigna et al 2011,

Table 3: Association of Low Back Pain and Physical Factors

\begin{tabular}{|c|c|c|c|}
\hline Physical Factors & LBP Mean (SD) & No-LBP Mean (SD) & p-value \\
\hline \multicolumn{4}{|c|}{ Practical exposure in hours $(n=194)$} \\
\hline Practical exposure & $209.8( \pm 244.9)$ & $132.2( \pm 207.8)$ & $0.02 *$ \\
\hline Theory exposure & $334.1( \pm 108.6)$ & $354.8( \pm 111.1)$ & 0.2 \\
\hline Total exposure & $1748.4( \pm 986.6)$ & $1439.7( \pm 1029.2)$ & $0.04 *$ \\
\hline \multicolumn{4}{|c|}{ PA's - pain experience $(n=176)$} \\
\hline PA's on L4 & $30.8 \%(20 / 65)$ & $11.7 \%(13 / 111)$ & $0.003^{*}$ \\
\hline PA's on L4 L & $41.5 \%(27 / 65)$ & $25.2 \%(28 / 111)$ & 0.07 \\
\hline PA's on L4 R & $47.7 \%(31 / 65)$ & $24.3 \%(27 / 111)$ & $0.003^{*}$ \\
\hline PA's on L5 & $43.1 \%(28 / 65)$ & $14.4 \%(16 / 111)$ & $<0.001 *$ \\
\hline PA's on L5 L & $47.7 \%(31 / 65)$ & $27.0 \%(30 / 111)$ & $0.02 *$ \\
\hline PA's on L5 R & $49.2 \%(32 / 65)$ & $28.8 \%(32 / 111)$ & $0.004^{*}$ \\
\hline \multicolumn{4}{|c|}{ TrA - successful recruitment $(n=176)$} \\
\hline TrA recruitment $-\mathrm{L}$ & $100 \%(65 / 65)$ & $98.2 \%(109 / 111)$ & 0.51 \\
\hline TrA recruitment $-\mathrm{R}$ & $98.5 \%(64 / 65)$ & $100 \%(111 / 111)$ & 0.31 \\
\hline \multicolumn{4}{|c|}{ LM CSA in $\mathrm{cm}^{2}(\mathrm{n}=170)$} \\
\hline LM CSA - L & $6.0( \pm 1.7)$ & $6.6( \pm 1.5)$ & $0.02 *$ \\
\hline LM CSA - R & $6.1( \pm 1.7)$ & $6.4( \pm 1.5)$ & 0.25 \\
\hline \multicolumn{4}{|l|}{ USI in $\mathrm{mm}(\mathrm{n}=170)$} \\
\hline Ol rest $-\mathrm{L}$ & $7.2( \pm 2.4)$ & $6.6( \pm 2.0)$ & 0.06 \\
\hline Ol rest $-\mathrm{R}$ & $7.4( \pm 2.1)$ & $6.7( \pm 1.9)$ & $0.02 *$ \\
\hline TrA rest $-\mathrm{L}$ & $4.4( \pm 0.9)$ & $4.3( \pm 1.3)$ & 0.62 \\
\hline TrA rest $-\mathrm{R}$ & $4.6( \pm 1.2)$ & $4.2( \pm 1.0)$ & $0.02 *$ \\
\hline TrA slide $-\mathrm{L}$ & $17.2( \pm 3.2)$ & $18.1( \pm 2.1)$ & $0.03 *$ \\
\hline TrA slide $-\mathrm{R}$ & $18.5( \pm 2.3)$ & $18.4( \pm 2.1)$ & 0.92 \\
\hline \multicolumn{4}{|c|}{ TrA Activity - \%change $(n=170)$} \\
\hline TrA activity - L & $55.2(32.8)$ & $46.6(39.4)$ & 0.15 \\
\hline TrA activity - R & $53.9(35.9)$ & $51.2(31.0)$ & 0.61 \\
\hline Ol activity - L & $8.9(17.7)$ & $10.2(18.6)$ & 0.66 \\
\hline Ol activity - R & $6.3(13.7)$ & $10.6(20.2)$ & 0.14 \\
\hline
\end{tabular}

* Factors associated with LBP (significance was set at $\mathrm{p}<0.05$ )

$\mathrm{CSA}=$ cross-sectional area, OI=oblique internal, OI activity=OI activity $=(\mathrm{OI}$ contracted-OI rest $) / O \mathrm{I}$ rest $\times 100, \mathrm{~L} 4=4^{\text {th }}$ lumbar vertebrae, $\mathrm{L} 5=5^{\text {th }}$ lumbar vertebrae, LBP $=$ low back pain, $\mathrm{LM}=$ lumbar multifidus, PA $=$ posterior-anterior mobilisation.

Total exposure $=$ practical and theory combined,

$\operatorname{Tr} A=$ transversus abdominis, $\operatorname{Tr} A$ activity $=(\operatorname{Tr} A$ contracted-TrA rest $) / \operatorname{Tr} A$ rest $x 100$. 
Steyl et al 2010, Karachi et al 2007, Nyland and Grimmer 2003). Compared to four South African cross-sectional prevalence studies that relate to whether undergraduate physiotherapy study is a risk factor for LBP (Falavigna et al 2011, Steyl et al 2010, Karachi et al 2007, Nyland and Grimmer 2003), the results of this study are lower; $73 \%(p=0.03)$ prevalence of LBP at one university followed by $61 \%$ and $57 \%$ at two other universities respectively (Karachi et al 2007). Methodological differences of the above studies compared to this study could have influenced results and possibly explain the differences in prevalence rates. The high prevalence

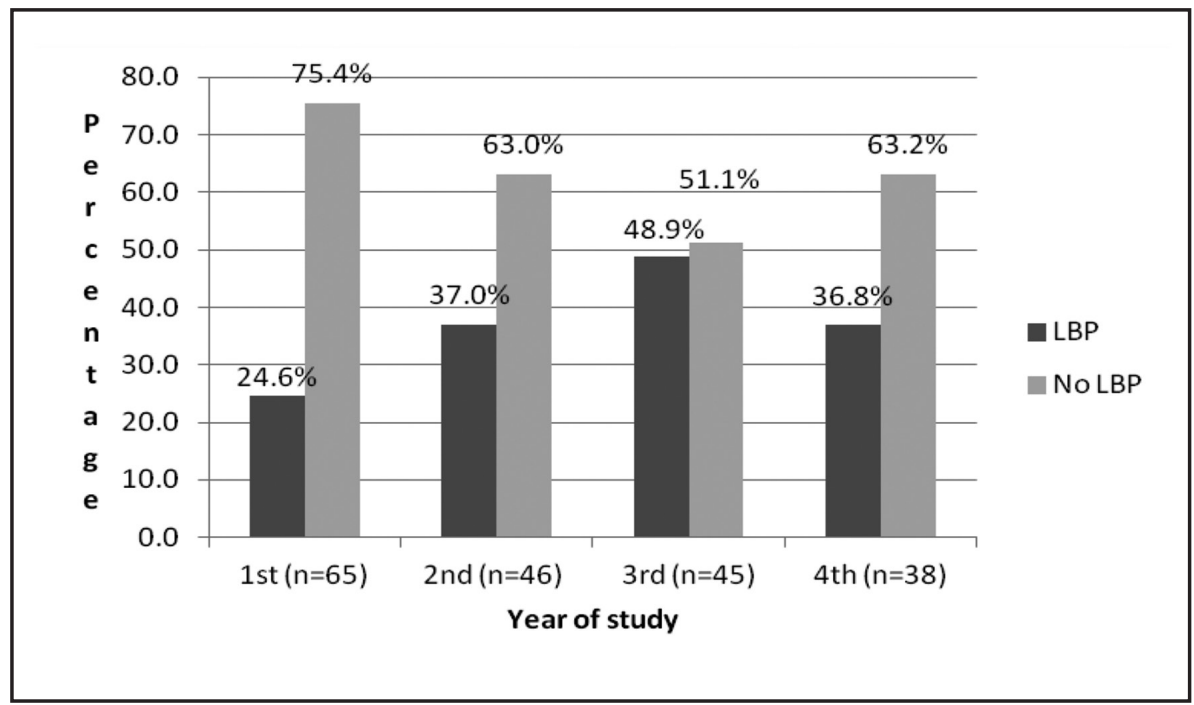

Figure 5: Prevalence of Low Back Pain by Year of Study $(n=194)$

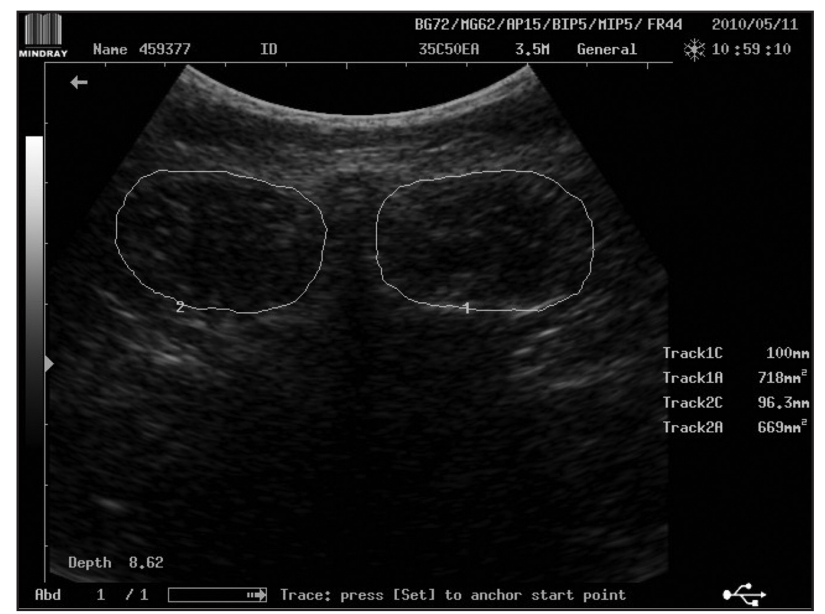

Figure 1: Image of Lumbar Multifidus at L4 with Ultrasound Imaging

In the above image the internal caliper of the ultrasound unit was used to trace the circumference. This was $100 \mathrm{~mm}(10 \mathrm{~cm})$ for the right side of the LM, from which a CSA of $718 \mathrm{~mm}^{2}\left(7.18 \mathrm{~cm}^{2}\right)$ is estimated. On the left side the circumference was $96.3 \mathrm{~mm}(9.63 \mathrm{~cm})$ and the CSA $669 \mathrm{~mm}^{2}\left(6.69 \mathrm{~cm}^{2}\right)$. During the recording of LM, the resting shape (oval) was also noted for qualitative analysis.

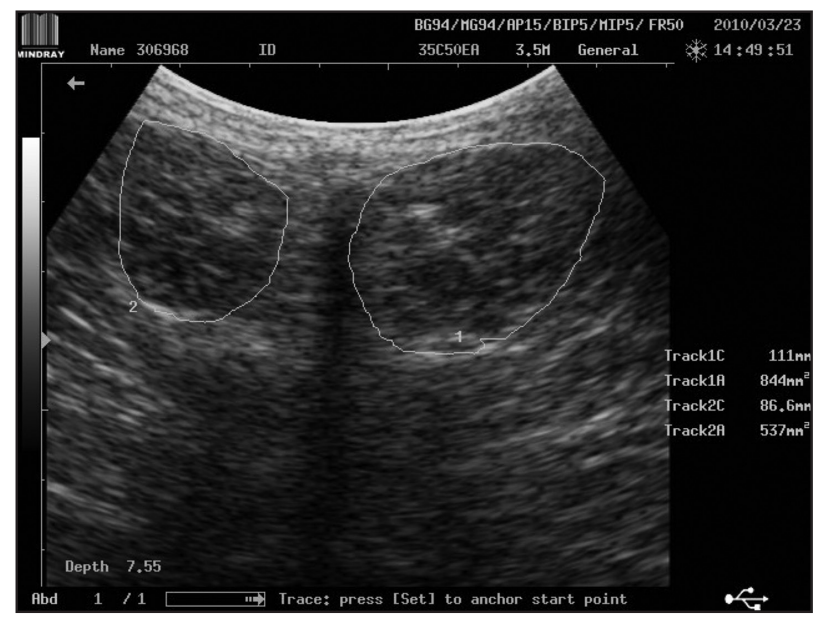

Figure 2: Left and Right Side of Lumbar Multifidus Asymmetrical with Ultrasound Imaging

A CSA of $537 \mathrm{~mm}^{2}\left(5.37 \mathrm{~cm}^{2}\right)$ on the left side and $844 \mathrm{~mm}^{2}\left(8.44 \mathrm{~cm}^{2}\right)$ on the right side were noted. The asymmetry indicate atrophy or pathology in the LM muscle.

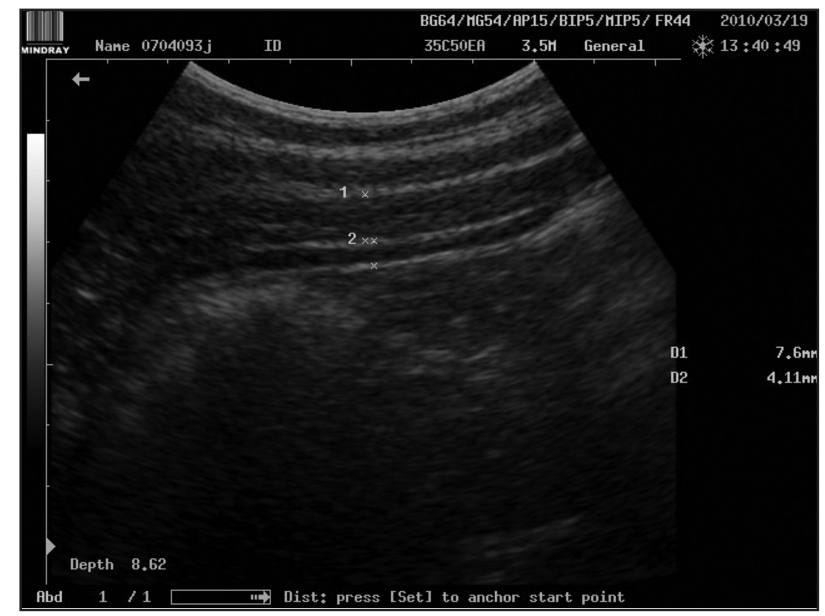

Figure 3: Resting Image of the Left Lateral Abdominal Wall The first measurement was taken at the end of respiration. The measurement at rest of OI (D1) was $7.6 \mathrm{~mm}$ and for $\operatorname{TrA}$ (D2) was $4.11 \mathrm{~mm}$.

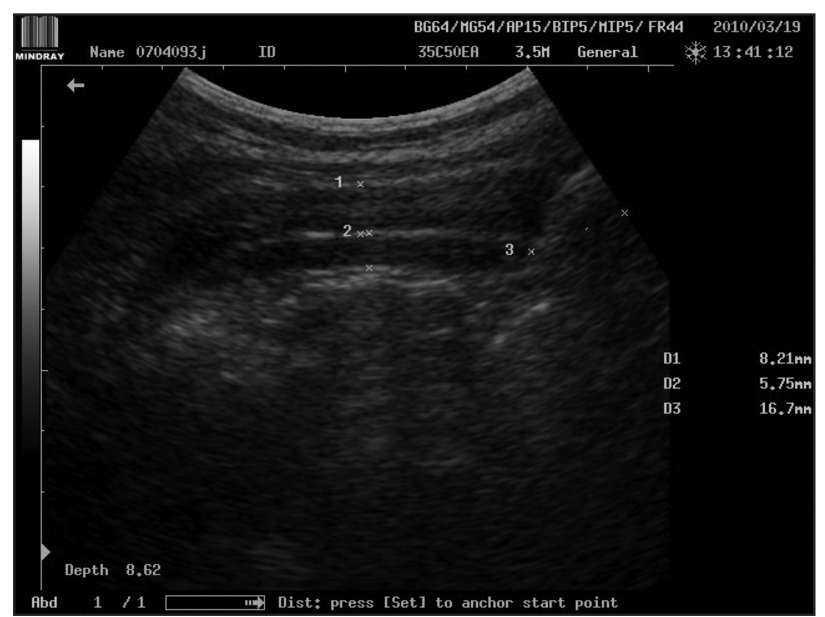

Figure 4: Contraction of the Abdominal Wall

For preferential activation of the $\operatorname{Tr} A$, an isolated contraction of the $\operatorname{Tr} A$ was expected. The increase in depth and lateral corseting were noted as the TrA slides under the $\mathrm{OI}$ and increases tension in the anterior TrA fascia. An increase was noted during contraction. The measurement of Ol was $8.21 \mathrm{~mm}$, for $\operatorname{TrA}$ was $5.75 \mathrm{~mm}$ and the TrA pull $1.67 \mathrm{~cm}$. 
among their respondents could be that questionnaires were mailed to the participants whereas in this study the questionnaires were completed in the presence of the first author. This could have created bias in the response to LBP absence or presence because those respondents without LBP may have been less likely to return their questionnaires. A higher percentage of physiotherapy students in this study completed questionnaires (93\%) compared to $72 \%$ in Karachi et al (2007) and 58\% in Nyland and Grimmer (2003). Despite proper training, information and incremental knowledge of the discipline of physiotherapy, students nevertheless injure themselves. This is a cause for concern as a career in physiotherapy places a huge physical demand on the body.

Of interest is a correlation between year of study, exposure to practical training and LBP. Inspite of proper training, information and incremental knowledge of the discipline of physiotherapy, students still injure themselves. One of the note worthy findings of this study was the apparent atrophy of LM on the left side $(p=0.02)$. This is supported by studies that also have found localised LM atrophy in participants with LBP (Hides et al 2008a). Studies have also shown that the atrophied LM does not recover spontaneously (Hides et al 1996) and the CSA of LM is reduced ipsilaterally on the side of symptoms (Hides et al 1996). According to the literature and the results of this study the left side was where most of the participants had pathology. Another related change following LBP affecting the muscles is the increase in intramuscular fat and fatigability (Hides et al 2008b). Hides et al (2008a) noted that fatty deposits or fibrous tissue infiltration and atrophy are common radiological findings. These morphological and physiological changes occur even when the individual has resumed work, sport and activity (Hides et al 2008b, Hides et al 1996).

LM muscle function and size are restored when specific training of the LM occurs at specific levels (Hides et al 2008b). This highlights the need to consider LM training as both a preventative and treatment measure for
LBP in physiotherapy students. Once LBP is present greater efforts should be made to act on the consequences by correcting muscle imbalances to restore function and size after pain and pathology (O’Sullivan 2005, Panjabi et al 1989). A physiotherapy student who has experienced LBP can prevent further damage and predisposition to LBP by addressing these specific muscles during training before qualifying and starting work as a physiotherapist. This could be included in a LBP prevention programme specifically targeted at students.

No differences were found with $\operatorname{Tr} A$ recruitment or activation in students with or without LBP. This is in contrast to other studies where Hodges and Richardson (1996) observed delayed and inefficient muscle activity (LM and TrA) in individuals with LBP. However in this study there were indeed differences in the thickness of $\operatorname{TrA}$ and OI when assessed by means of USI at rest in those with and those without LBP $(p=0.02)$. $\mathrm{OI}$ and $\operatorname{Tr} \mathrm{A}$ on the right were thicker at rest in LBP participants, while TrA slide was found to be less on the left side with LBP which indicates less recruitment on that side. Lumbar pathology may have resulted in these adaptations to the lumbar musculature. It therefore follows that on the right side anteriorly, students are affected by pain shown by the larger measurements during rest of $\operatorname{TrA}$ and OI to provide cross-over stability for the smaller measurement of the CSA of LM on the left posteriorly. As with the LM a detailed and specific assessment of the students abdominal muscles could be included in a LBP prevention programme and could be an opportunity for student learning of specific assessments of abdominal muscles whose dysfunction contributes to LBP.

The development of LBP contributes to poor movement habits, causing muscle imbalances. These muscle imbalances may increase strain on nerve tissue, muscles and the joints which, if overloaded, cause either additional pathology or pain (Comerford and Mottram 2001b). In response to LBP, motor control deficit has been found to cause poor motor control of the spine (O’Sullivan 2005). Neuromuscular responses may follow, including seg- mental reflex inhibition, altered recruitment patterns and timing of the LM and $\operatorname{TrA}$. The stability of the spine and neural structures are affected, resulting in additional LBP and an abnormal posture (O'Sullivan 2005). O'Sullivan (2005) noted that either an excess or deficit in spinal stability affects functional stability. Muscles may become fibrotic and shorten, losing extensibility and increasing instability of the spine (Panjabi et al 1989). The loss in size of LM and TrA are not regained spontaneously upon resolution of pain and disability, possibly causing further injury of the spine and recurrences of LBP (Hides et al 1996). The cycle of LBP and recurrences will continue unless these factors are addressed.

LBP appears to be a real issue for physiotherapy students during their training. A physiotherapy student who has experienced LBP can mitigate further damage and predisposition to LBP by addressing muscle activation and imbalances of TrA, LM and OI as an early intervention programme before qualifying and starting work as a physiotherapist. There should also be more emphasis on prevention of LBP in students without a prior history, and the development of risk assessment tools that will help to identify and address specific educational and clinical exposure hazards for the students. Programmes on manual handling, ergonomics and kinetic handling could be implemented in order to make students more aware and better equipped to protect themselves.

The authors recommend that all the year groups should be tested at the same time of year, preferably at the end of the year. The students would have been exposed to the different requirements for each year and thus prevalence of LBP at the end of each year would be more consistent when the students are tested, left or right handedness could be added to the questionnaire to allow for lateralisation of findings in students with LBP. Probable LM atrophy on the left side and preponderance of muscle thickness differences on the right may have been explained by left or right handedness. This hypothesis is supported by Sung et al (2004) who noted delayed back muscle response time on the 
non-dominant side. In the physical assessment, posture was also not evaluated to confirm the muscle imbalances and again this could be included in future studies. A cross sectional study cannot establish causal relationships and a prospective study following students from first to fourth year with an appropriate intervention could now follow with the results from this study providing useful preliminary data.

\section{CONCLUSION}

Associations with LBP amongst physiotherapy students were hours of practical exposure, posterior-anterior mobilisations on L4 and L5 centrally and unilaterally, left LM CSA, right OI and right TrA thickness at rest, as well as the pull of the TrA during contraction on the left. It is important to adequately train and make the students aware of LBP injuries in their first year of the physiotherapy programme, therefore empowering them to reduce the prevalence of LBP. Physiotherapists may be at lower risk of developing LBP as a product of musculoskeletal dysfunction if they were able to enter the profession with an awareness of the factors associated with the LBP while studying at an undergraduate level.

\section{REFERENCES}

Comerford M, Mottram S 2001.a Functional stability re-training: principles and strategies for managing mechanical dysfunction. Manual Therapy 6:3-14

Comerford M, Mottram S 2001.b Movement and stability dysfunction-contemporary developments. Manual Therapy 6:15-26

Dankaerts W, O’Sullivan P, Burnett A, Straker L, Davey P, Gupta R 2009. Discriminating healthy controls and two clinical subgroups of nonspecific chronic low back pain patients using trunk muscle activation and lumbosacral kinematics of postures and movements: a statistical classification model. Spine 34:1610

Falavigna A, Teles AR, Mazzocchin T, De Braga GL, Kleber FD, Barreto F, Santin JT, Barazzetti D, Lazzaretti L, Steiner B, Beckenkamp NL 2011. Increased prevalence of low back pain among physiotherapy students compared to medical students. European Spine Journal 20:500-505
Hides J, Gilmore C, Stanton W, Bohlscheid E 2008.a Multifidus size and symmetry among chronic LBP and healthy asymptomatic subjects. Manual Therapy 13:43-49

Hides JA, Richardson CA, Jull GA 1996. Multifidus muscle recovery is not automatic after resolution of acute, first-episode low back pain. Spine 21:2763-2769

Hides JA, Stanton WR, McMahon S, Sims K, Richardson CA 2008.b Effect of stabilization training on multifidus muscle cross-sectional area among young elite cricketers with low back pain. Journal of Orthopaedic and Sports Physical Therapy 38:101-108

Hodges P, Pengel L, Herbert R, Gandevia S 2003. Measurement of muscle contraction with ultrasound imaging. Muscle Nerve 27:682-692

Hodges PW, Richardson CA 1996. Inefficient muscular stabilization of the lumbar spine associated with low back pain: a motor control evaluation of transversus abdominis. Spine 21: 2640-2650

Karachi F, S. B, Cameron A, Honey L, McDougall $\mathrm{K} 2$ 2007. The prevalence of lower back pain amongst undergraduate physiotherapy students in the Western Cape. (unpublished data)

Maitland G, Banks K, English K, Hengeveld E 2001. Maitland's Vertebral Manipulation 6th ed. ppl 351-355. Alden Press, Oxford

Nyland LJ, Grimmer KA 2003. Is undergraduate physiotherapy study a risk factor for low back pain? A prevalence study of LBP in physiotherapy students. BMC Musculoskelet Disorders 4:22

O’Sullivan P 2005. Diagnosis and classification of chronic low back pain disorders: maladaptive movement and motor control impairments as underlying mechanism. Manual Therapy 10: 242-255

Panjabi M, Abumi K, Duranceau J, Oxland T 1989. Spinal stability and intersegmental muscle forces: a biomechanical model. Spine 14:194-200

Shum GL, Crosbie J, Lee RY 2010. Back pain is associated with changes in loading pattern throughout forward and backward bending. Spine (Phila Pa 1976) 35:E1472-1478

Steyl T, Constance I, Bogopa M, Engelbrecht L, Ramkisson K, Opperman T 2010. The relationship between musculoskeletal dysfunction and physical activity participation of undergraduate physiotherapy students at the University of the Western Cape. University of the Western Cape
Stokes M, Rankin G, Newham D 2005. Ultrasound imaging of lumbar multifidus muscle: normal reference ranges for measurements and practical guidance on the technique. Manual Therapy 10:116-126

Sung PS, Spratt KF, Wilder DG 2004. A possible methodological flaw in comparing dominant and nondominant sided lumbar spine muscle responses without simultaneously considering hand dominance. Spine 29:1914-1922

Teyhen DS, Williamson JN, Carlson NH, Suttles ST, O'Laughlin SJ, Whittaker JL, Goffar SL, Childs JD 2009. Ultrasound characteristics of the deep abdominal muscles during the active straight leg raise test. Archives of physical medicine and rehabilitation 90:761-767 